\title{
Open-Label Taste-Testing Study to Evaluate the Acceptability of Both Strawberry-Flavored and Orange-Flavored Amylmetacresol/2,4-Dichlorobenzyl Alcohol Throat Lozenges in Healthy Children
}

\author{
Alex Thompson · Sandie Reader • Emma Field • \\ Adrian Shephard
}

Published online: 16 April 2013

(C) The Author(s) 2013. This article is published with open access at Springerlink.com

\begin{abstract}
Background Acute sore throat (pharyngitis) is one of the most common illnesses for which children are seen by primary care physicians. Most cases are caused by viruses and are benign and self-limiting. Clinically proven, overthe-counter throat lozenges provide rapid and effective relief of acute sore throat symptoms, and are increasingly important in self-management of this condition.

Objective The purpose of this study (International Standard Randomized Controlled Trial Number: ISRCTN34958871) was to evaluate the acceptability of two licensed, commercially available sore throat lozenges containing amylmetacresol and 2,4-dichlorobenzyl (AMC/ DCBA) - one strawberry flavored and the other orange flavored-in healthy children.

Study design This was an open-label, single-dose, crossover, taste-testing study in children recruited via a clinical database and advertisements over a 3.5-week period.

Setting Potentially eligible participants were invited to attend the taste-testing session at a clinic.
\end{abstract}

\footnotetext{
A. Thompson $(\square)$

Aspect Clinical, 4a Bye Street, Ledbury,

Herefordshire HR8 2AA, UK

e-mail: alex.thompson@aspectclinical.com

S. Reader

Clearcut Clinical Consulting Ltd, 37 Wollaton Vale,

Nottingham, UK

E. Field

Reckitt Benckiser Healthcare Ltd, Dansom Lane, Hull,

Yorkshire, UK

A. Shephard

Reckitt Benckiser Healthcare Ltd, 103-105 Bath Road, Slough,

Berkshire, UK
}

Participants At the screening session, which took place either before or on the day of taste testing, details of relevant medical history, medication, and demographics were recorded. Of the 108 screened subjects, 102 were recruited. These were healthy male and female children aged 6-12 years.

Intervention Each child cleansed their palate with water and water biscuits before tasting a strawberry-flavored lozenge (Strepsils ${ }^{\circledR}$ strawberry sugar free, Reckitt Benckiser Healthcare Limited, Nottingham, UK; PL 00063/0395), which was sucked for 1 minute and then expelled. The orange-flavored lozenge (Strepsils ${ }^{\circledR}$ orange with vitamin C, Reckitt Benckiser Healthcare Limited, Nottingham, UK; PL 016242152) was tasted at least 15 minutes later following further cleansing of the palate. The spontaneous reaction of the child on tasting each lozenge was observed and recorded. Subjects were asked to indicate their liking for each lozenge, using a 7-point hedonic facial scale, and were required to answer a series of questions relating to what they liked and disliked about the taste and the feel of the lozenge in the mouth and throat. The primary endpoint was the proportion of subjects with a hedonic facial score of $>4$. Secondary endpoints included the spontaneous reaction of the child on tasting the lozenge and responses to questions related to taste.

Results The taste of the lozenge was scored $>4$ (i.e. 'good', 'really good', or 'super good') by $85.3 \%$ of subjects for the strawberry flavor and $49.0 \%$ for the orange flavor $(p<0.0001)$. The mean (standard deviation) score was 5.72 (1) for the strawberry-flavored lozenge and 4.35 (2) for the orange-flavored lozenge. The proportion of subjects willing to take the lozenge again was $94 \%$ for the strawberry flavor and $56 \%$ for the orange flavor.

Conclusions Strawberry-flavored AMC/DCBA lozenges were liked by, and acceptable to, the majority of the 
children. AMC/DCBA orange-flavored lozenges were also liked by, and acceptable to, approximately half the children. Overall, both strawberry and orange would be suitable flavors for lozenges intended for children when they suffer from sore throat.

\section{Introduction}

Acute sore throat (pharyngitis) is one of the most common illnesses for which children and their parents visit primary care physicians [1]. For example, in the ambulatory setting, acute pharyngitis accounts for around $1 \%$ of primary care visits [2]. Most cases (up to $80 \%$ ) are caused by viruses and are benign and self-limiting [3]. However, bacteria (e.g. group A beta-hemolytic streptococci) are another common cause, particularly among children [4].

The diagnosis of pharyngitis must distinguish children with viral pharyngitis, who would not benefit from antibiotic therapy, from those children with group A betahemolytic streptococcal pharyngitis, for whom antibiotics are appropriate [1]. Making this distinction is crucial in attempting to minimize the unnecessary use of antimicrobial agents in children and providing suitable symptomatic relief. The absence of fever or the presence of clinical features such as conjunctivitis, cough, or hoarseness, suggest a viral etiology [1].

The clinical manifestations of acute sore throat are related to inflammation of the pharynx and/or tonsils, and include pain, redness, heat, and swelling [5, 6]. Despite the fact that antibiotics are still often requested and prescribed for acute sore throat, many patients (adults and children) consult their primary care physician to establish the cause of the symptoms, to obtain pain relief, and for information on the course of the disease [7, 8]. Furthermore, because the majority of sore throats are caused by viruses and not bacteria, antibiotics are generally ineffective and not recommended by clinical bodies for primary treatment of sore throat [9]. Instead, clinically proven over-the-counter (OTC) medications, which provide rapid and effective relief of symptoms of acute sore throat, regardless of cause, are increasingly important in the self-management of this condition.

Throat lozenges containing amylmetacresol (AMC) and 2,4-dichlorobenzyl alcohol (DCBA), which possess antibacterial, antiviral, and local anesthetic properties, provide symptomatic relief of sore throat $[6,10]$. They are licensed for OTC use in the UK and around the world for adults and children for the symptomatic relief of mouth and throat infections [11]. Safety profiles are well established, and in some countries the lozenges have been used for over 50 years.

Lozenges containing AMC/DCBA have been studied in several clinical trials conducted in adults and have demonstrated significant analgesic, functional, sensorial, and psychological effects from as early as $1-5$ minutes and lasting up to $2 \mathrm{~h}$ post-dose $[5,12,13]$. For example, studies have shown that patients taking AMC/DCBA throat lozenges reported experiencing relief from their throat soreness and sore throat pain but also soothing and coating effects [5, 14]. These lozenges have also been shown to be effective for the symptomatic treatment of sore throat in children aged $>5$ years with acute and aggravated chronic pharyngitis [15].

A primary consideration for the development of a pediatric formulation is the acceptability to children [16]. Many investigators cite palatability as an important factor in medication adherence and completion of therapy in children, although formal studies are lacking [17]. Little direct evidence exists to show that poor palatability decreases adherence; however, it is not unreasonable to assume that a more palatable medication is easier to administer to infants and young children. Previous taste testing in children has shown that they generally prefer sweet preparations with fruit flavors [18]. National favorites are bubble gum and grape in the USA, citrus and red berries in Europe, and liquorice in Scandinavia [16].

The hedonic facial scale, which uses a pictorial scale of facial expressions, has been commonly employed in determining the acceptability of medications to children [18]. Compared with spontaneous verbal judgment, this method has the advantage of being more standardized. Studies have shown that children aged as young as 4 years can understand and use this scale to indicate whether a substance tastes pleasant and is therefore acceptable [18]. This scale has previously been used to evaluate the acceptability of a wide range of medications among children, including steroid preparations [19], antibiotics [20-22], calcium and vitamin $\mathrm{D}_{3}[23,24]$, ondansetron [25], and lansoprazole [26, 27].

The purpose of this study was to evaluate the acceptability of two licensed, commercially available throat lozenges containing AMC/DCBA, one strawberry and the other orange flavored, in healthy children aged 6-12 years, taken sequentially on the taste-testing day. Taste was assessed using the 7-point hedonic facial score, which was the primary measure of acceptability, as well as spontaneous reaction and verbal responses to questions relating to palatability, flavor, and the feel of the lozenge in the mouth.

\section{Methods}

\subsection{Study Design}

This was an open-label, single-dose, crossover, taste-testing study in children to investigate the acceptability of two 
different flavors of AMC/DCBA lozenges. It was conducted in accordance with the Declaration of Helsinki [28] and was reviewed by the Reading Independent Ethics Committee (Reading, Berkshire, UK). The International Standard Randomized Controlled Trial Number is ISRCTN34958871.

As this study was designed to assess the acceptability of the two lozenge flavors in absolute rather than comparative terms, blinding was not considered necessary given that flavor acceptability, as opposed to efficacy, was the parameter of interest and would have been difficult to achieve as the products taste different.

The subject population comprised healthy children aged 6-12 years, the age range of the target population. Although no formal sample size calculation was performed, 100 children tasting both samples were believed to be an appropriate sample number to evaluate. It was estimated that 120 subjects would need to be screened in order to achieve this.

\subsection{Subject Selection}

Healthy males and females (aged 6-12 years) were recruited from a clinical trial company's database and via advertisements over a 3.5-week period. Parents provided written informed consent for the participation of their child in the study, and the child voluntarily wrote or marked their name on the assent form. Subjects were screened either before or on the day of taste testing, and details of any relevant medical history, medication, and demographics were recorded.

Subjects were excluded if they had a history of hereditary fructose intolerance; sensitivity to an analgesic medication, its ingredients or related products; or any previous history of allergy or known intolerance to AMC, DCBA, or any colouring, flavoring, preservative, sweetener, or surfactant. Other exclusion criteria were a history of hepatic or renal impairment, cardiac disease, high blood pressure, asthma, gastrointestinal disorders, respiratory infection, or any other condition that could have affected the subjects' perception of taste. Subjects were also excluded from enrolment on the taste-testing day if they had taken prescription medications during the previous 7 days, used analgesics or anesthetics, consumed food or drink that may have affected their perception of taste (e.g. highly spiced meals or mint- or menthol-based products) on the testing day, or used non-prescription medication within $4 \mathrm{~h}$ prior to taste testing. Other restrictions on the taste-testing day were the presence of a mouth ulcer or dental work carried out on that day. The taste-testing day was to be rescheduled for subjects who met any one of these restriction criteria.

\subsection{Treatments}

Before receiving a lozenge, each subject cleansed their palate with water and water biscuits. The subjects received a single strawberry-flavored, sugar-free AMC/DCBA lozenge (Strepsils ${ }^{\circledR}$ strawberry sugar free, Reckitt Benckiser Healthcare Limited, Nottingham, UK; PL 00063/0395) followed at least 15 minutes later by a single orange-flavored, colour-free AMC/DCBA lozenge (Strepsils ${ }^{\circledR}$ orange with vitamin C, Reckitt Benckiser Healthcare Limited, Nottingham, UK; PL 016242152). Each lozenge was sucked for 1 minute and then expelled. Both lozenges contained $0.6 \mathrm{mg}$ AMC and $1.2 \mathrm{mg}$ DCBA. In addition, the orange-flavored colour-free lozenge contained $100 \mathrm{mg}$ vitamin $\mathrm{C}$ as sodium ascorbate/ascorbic acid. Questions relating to the lozenges' palatability were then asked after each lozenge was spat out.

During protocol development, there were concerns that if the orange-flavored lozenge was tasted first, the strong orange and menthol flavor might affect the subsequent perception of the strawberry flavor, even after the required 15-minute interval and cleansing of the palate. Therefore, the strawberry-flavored lozenge was tasted first by all subjects. This was deemed acceptable given that the purpose of the study was to assess the acceptability of each flavor and not to compare the acceptability of the two flavors. The 15-minutes period between tasting the samples was considered appropriate in terms of maximizing subject compliance. A previous study has shown that complete lozenge dissolution takes approximately 6.77 minutes [29]. As the children in this study were only required to suck each lozenge for 1 minutes, they were not exposed to more than a standard dose (AMC $0.0022 \mathrm{mg} / \mathrm{mL}$ [standard deviation (SD) 0.0012] and DCBA $0.0097 \mathrm{mg} / \mathrm{mL}$ [SD 0.0040]).

\subsection{Acceptability Assessments and Endpoints}

Assessments on the taste-testing day were designed to evaluate the acceptability of both flavors to the children. During the taste-testing session, children were first asked what they would like their medication to taste of. Subjects were asked to indicate their liking for each lozenge, using a 7-point hedonic facial scale (Fig. 1), which included the following scores: $1=$ super bad; $2=$ really bad; $3=$ bad; $4=$ may be good $/ \mathrm{may}$ be bad; $5=$ good; $6=$ really good; $7=$ super good. After expelling the lozenge, the subjects were asked a series of questions relating to the taste and feel of the lozenge in the mouth and throat.

The primary endpoint was the percentage of children who rated each lozenge with a score of $>4$ on the 7-point hedonic facial scale, together with descriptive summary statistics (mean, SD, median, minimum, maximum) of the hedonic facial scale scores. 


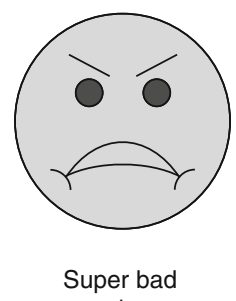

1

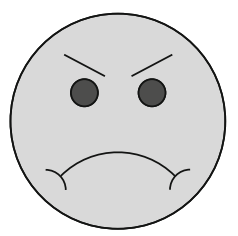

Really bad

2

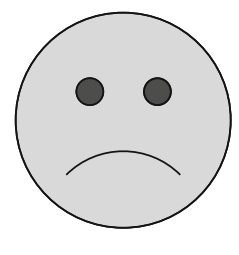

Bad

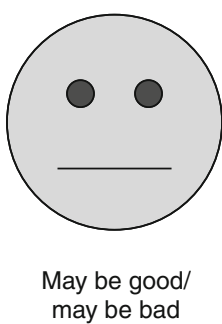

4

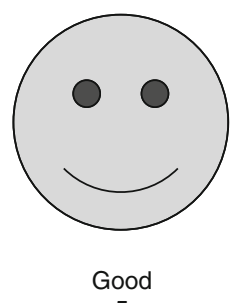

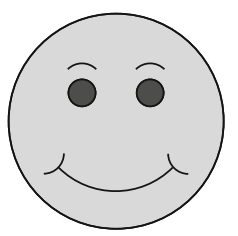

Really good

6

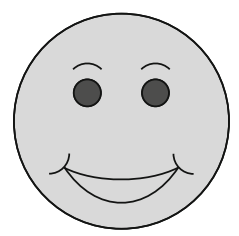

Super good

7

Fig. 1 The 7-point hedonic facial scale for assessment of acceptability [16]

Secondary endpoints included the observed spontaneous reaction to putting the lozenge in the subject's mouth (based on whether the subjects sucked the lozenge for 1 minute or spat it out), the flavor perceived by the subjects in response to the question "What does the medicine taste of?", and the subjects' responses to a series of questions about what they liked and disliked about the taste.

No efficacy assessments were conducted in this study. Assessment of safety included analysis of any adverse events (AEs) spontaneously mentioned by the subjects after they had received each flavor of lozenge.

\subsection{Statistical Methods}

For the primary endpoint, the proportion of subjects who had a hedonic facial score of $>4$ (i.e., 5-7) was presented together with the $95 \%$ confidence interval (CI), for each lozenge. For the secondary endpoints, descriptive summary statistics of the hedonic facial scale score for each lozenge were presented together with the $95 \%$ CI for the mean score. The number of times the sample was retained for 1 minute/spat out and responses to questions relating to taste were presented in the listings and summarized descriptively.

\section{Results}

A total of 108 subjects were screened, of whom 103 were eligible and entered the study (59 males; 44 females), with a mean age of 9 years (range 6-12 years). Medical history was reported for 19 subjects. One subject withdrew before tasting the first sample. A total of 102 subjects completed the study, tasting both samples, and were included in the analyses.

\subsection{Acceptability Analyses}

In response to the question "If you could choose the taste of your medicine, what would it taste of?", $44 \%$ of subjects indicated their preference would be strawberry/ strawberries, $11 \%$ chocolate, and $7 \%$ orange. For the
Table 1 Proportion of subjects selecting each score on a 7-point hedonic facial scale (primary endpoint)

Percentage of subjects selecting each score $^{\mathrm{a}}$

Strawberry-flavored Orange-flavored lozenge $(n=102) \quad$ lozenge $(n=102)$

Score

1: Super bad $\quad 2.0 \quad 9.8$

2: Really bad $\quad 1.0 \quad 5.9$

3: Bad $\quad 1.0 \quad 12.7$

4: May be good/may $\quad 10.8 \quad 22.5$ be bad

5: Good $\quad 17.6 \quad 22.5$

6: Really good $\quad 40.2 \quad 12.7$

$\begin{array}{lll}7: \text { Super good } & 27.5 & 13.7\end{array}$

Percentage [95 \% CI] of 85.3 [74.8-92.2] 49.0 [39.3-58.7] subjects selecting a score $>4$

$p$ value for difference $\quad<0.0001$

between treatments

${ }^{\text {a }}$ Numbers may not total $100 \%$, because of rounding CI confidence interval

primary endpoint, $85.3 \%$ of subjects rated the strawberry lozenge with a score of $>4$ and $49.0 \%$ rated the orangeflavored lozenge with a score of $>4 \quad(p<0.0001)$ (Table 1). The mean (SD) score was 5.72 (1) for the strawberry-flavored lozenge and 4.35 (2) for the orangeflavored lozenge (Table 2).

No subject spontaneously rejected either lozenge or spat it out before being required to do so. When asked directly, the proportion of subjects who had wanted to take the lozenge out of their mouth was $17 \%$ for the strawberry flavor and $46 \%$ for the orange flavor. The proportion of these subjects who wanted to remove the lozenge and who also rated the lozenges as 'super bad'/'really bad', or 'bad' was $4 \%$ for strawberry and $26.5 \%$ for orange. The proportion of subjects answering "yes" to the question "Would you be happy to take it again?" was $94 \%$ for the strawberry lozenge and $56 \%$ for the orange lozenge. The most common reason for not wishing to take the orange lozenge again was 
Table 2 Descriptive summary statistics of the 7-point hedonic facial scale for all subjects (primary endpoint)

\begin{tabular}{lll}
\hline & \multicolumn{2}{l}{ Hedonic facial scale score } \\
\cline { 2 - 3 } & $\begin{array}{l}\text { Strawberry-flavored } \\
\text { lozenge }(n=102)\end{array}$ & $\begin{array}{l}\text { Orange-flavored } \\
\text { lozenge }(n=102)\end{array}$ \\
\hline Mean scores in different age groups & \\
6 years $[n=13]$ & 6.15 & 4.62 \\
7 years $[n=6]$ & 5.33 & 4.33 \\
8 years $[n=16]^{\mathrm{a}}$ & 5.60 & 3.93 \\
9 years $[n=20]$ & 5.75 & 3.90 \\
10 years $[n=15]$ & 5.20 & 4.87 \\
11 years $[n=14]$ & 6.07 & 4.71 \\
12 years $[n=19]$ & 5.74 & 4.32 \\
Overall scores & & \\
Mean & 5.72 & 4.35 \\
Median & 6 & 4 \\
Maximum & 7 & 7 \\
Minimum & 1 & 1 \\
SD & 1 & 2 \\
SEM & 0.12 & 0.18 \\
UCL & 6 & 5 \\
LCL & 5 & 4 \\
\hline
\end{tabular}

${ }^{a}$ One subject withdrew from the study before tasting either lozenge $S D$ standard deviation, SEM standard error of the mean, $U C L$ upper confidence limit, $L C L$ lower confidence limit

that it tasted "sour" (13\% of subjects). The proportion of subjects answering "yes" to the question "Would you be happy for Mum or Dad to give you this flavor medicine when you get a sore throat?" was $86 \%$ for the strawberryflavored lozenge and $55 \%$ for the orange-flavored lozenge. When asked the specific question "Did your mouth feel soothed?", a high proportion of subjects taking either the strawberry $(87 \%)$ or orange $(67 \%)$ lozenge reported that their throat had been soothed by the lozenge.
When the subjects were asked how the medicine had made their throat feel, their responses were broadly similar for both lozenges; the proportion of subjects whose throat felt "normal", whose throat felt "not different", or who did not know was 41 and $32 \%$ for the strawberry- and orange-flavored lozenges, respectively (Table 3 ). These results were expected, as the study was conducted in healthy volunteers.

No AEs were reported in this study, which is in accordance with the well-established safety profile of $\mathrm{AMC} /$ DCBA lozenges.

\section{Discussion}

The palatability of medications, particularly those taken orally, is an important factor in determining medication adherence and completion of drug therapy in young children, and should be an important part in the development process of new pediatric formulations [16]. This tastetesting study was based on well-recognised and accepted techniques for evaluating the taste of pediatric formulations $[30,31]$ and, based on the results of this study, would seem to be applicable to lozenge formulations.

In this study, a high proportion $(85.3 \%)$ of the children rated the strawberry-flavored lozenge as tasting 'good', 'really good', or 'super good'. These results are consistent with those of other studies of strawberry-flavored medications in children. For example, in an assessment of antibiotics, Angelilli et al. [22] found that a strawberry-flavored cefixime preparation was most commonly rated as the best tasting when compared with cherry-, bubble gum- and banana-flavored preparations in children aged 5-8 years. In a further study, strawberry-flavored lansoprazole suspension and tablets were preferred by over $90 \%$ of children (5-11 years), compared with peppermint-flavored ranitidine syrup [26, 27]. In another study, significantly more

Table 3 Subjects' responses to the questions "How did it make your mouth feel?" and "How did it make your throat feel?"

\begin{tabular}{|c|c|c|c|c|}
\hline \multirow[t]{3}{*}{ Comment } & \multicolumn{4}{|c|}{ Percentage of subjects selecting each score } \\
\hline & \multicolumn{2}{|l|}{ Mouth } & \multicolumn{2}{|l|}{ Throat } \\
\hline & $\begin{array}{l}\text { Strawberry-flavored lozenge } \\
(n=102)\end{array}$ & $\begin{array}{l}\text { Orange-flavored lozenge } \\
(n=102)\end{array}$ & $\begin{array}{l}\text { Strawberry-flavored lozenge } \\
(n=102)\end{array}$ & $\begin{array}{l}\text { Orange-flavored lozenge } \\
(n=102)\end{array}$ \\
\hline $\begin{array}{l}\text { Normal/not different/ } \\
\text { don't know }\end{array}$ & 36 & 6 & 41 & 32 \\
\hline Generally positive & 19 & 9 & 26 & 19 \\
\hline $\begin{array}{l}\text { Smooth/soothed/soft/ } \\
\text { calm }\end{array}$ & 5 & 3 & 16 & 14 \\
\hline Tingly/numb & 17 & 24 & 6 & 12 \\
\hline Generally negative & 4 & 14 & 4 & 6 \\
\hline Hot & 3 & 12 & NR & NR \\
\hline
\end{tabular}

$N R$ not reported 
children (aged 3-12 years) preferred strawberry-flavored ondansetron syrup to grape-flavored syrup [25].

However, one strawberry flavor or other flavor may taste quite different to another, depending on the flavor supplier, the medicinal format of the product containing the flavor, or other ingredients with a taste within the formulation, such as the active ingredients. We demonstrated that these particular flavors in AMC/DCBA lozenges were liked by a majority of children within this study.

A previous study conducted in children aged 4-7 years found that sweetness was the most important flavor characteristic for a medication, together with the effective masking of any bitter taste of the active ingredients [32]. In addition, red fruit (strawberry/raspberry) flavor was found to be the most acceptable to children and although the majority readily accepted citrus flavors, they did not prefer them. Citrus flavor was preferred to banana flavor by fewer children when compared with formulations containing calcium and vitamin $\mathrm{D}_{3}$ [24]. Therefore, in our study, the sourness of the flavor may have been a factor, with the lower absolute palatability of the orange-flavored lozenge being in line with the major dislike reported in this study.

The open-label, uncontrolled design of the study was appropriate given that the objective was to investigate absolute rather than comparative acceptability of the samples. The order in which the samples were tasted was not randomized because of the possibility that the menthol in the orange-flavored lozenge could carry over and affect the taste of the strawberry-flavored lozenge, as menthol in lozenges has been demonstrated to exert effects that are still experienced 30 minutes after consumption [33]. Therefore, the strawberry-flavored lozenge was taken before the orange-flavored lozenge.

A potential limitation of this study is that it was conducted in healthy children, and thus the results may not necessarily be translated directly to children with acute sore throat, whose perceptions of flavor may be affected by symptoms of a cold [34]. However, since the main purpose of this study was to evaluate the general acceptability of the two flavored lozenges in absolute rather than comparative terms, and the impact of symptoms of a cold on perception of flavor may differ between subjects, the inclusion of healthy children in this study is considered reasonable. Future work may be warranted involving children with symptoms of upper respiratory tract infection/sore throat.

It is also possible that the order in which the lozenges were tasted (strawberry then orange) had a bearing on the vocabulary used in the responses given to the question asking what the subjects disliked about the flavor. After being asked general questions about what they liked/disliked about the strawberry lozenge, subjects were then asked more specific questions such as "Do you think it tasted sour [like a lemon]?". The subjects then tasted the orange-flavored lozenge and were asked the same questions in the same order. Therefore, when subjects were voluntarily describing the taste of the strawberry-flavored lozenge, they had not yet been presented with the words "sour" or "lemon", but when they were describing the orange-flavored lozenge, they had already been given the association of "sour" and "lemon".

\section{Conclusions}

Strawberry-flavored sugar-free AMC/DCBA lozenges were liked by, and acceptable to, the majority of the children in this study; this flavor preference is in line with previous children's medicine studies in Europe. Orange-flavored colour-free AMC/DCBA lozenges with vitamin $\mathrm{C}$ were liked by, and acceptable to, approximately half of the children, and older children (10-12 years) found them more acceptable than 6- to 10-year-olds did. Overall, both strawberry and orange would be suitable flavors for lozenges intended for children when they suffer from sore throat.

Acknowledgements This study was funded by Reckitt Benckiser Healthcare Ltd, UK. Editorial assistance for the development of this article was provided by Elements Communications Ltd, UK, supported by Reckitt Benckiser Healthcare Ltd, UK.

Author Contributions Alex Thompson contributed to the acquisition, analysis, and interpretation of data. Sandie Reader contributed to the writing of the clinical study report. Emma Field contributed to the writing of the study protocol and clinical study report. Adrian Shephard contributed to the concept development of the study and the study protocol and reviewing of the clinical study report. All authors were involved in drafting, reviewing, and final approval of the manuscript.

Conflict of Interest Alex Thompson is employed by Aspect Clinical, who were paid by Reckitt Benckiser to conduct the study. Dr Thompson received no direct payments to conduct the study. Sandie Reader has received payments from Reckitt Benckiser for freelance clinical project management and medical writing in the past 5 years, and was paid to write the clinical study report on which this manuscript is based. Emma Field and Adrian Shephard are employees of Reckitt Benckiser.

Open Access This article is distributed under the terms of the Creative Commons Attribution Noncommercial License which permits any noncommercial use, distribution, and reproduction in any medium, provided the original author(s) and the source are credited. The exclusive right to any commercial use of the article is with Springer.

\section{References}

1. Gerber MA. Diagnosis and treatment of pharyngitis in children. Pediatr Clin North Am. 2005;52(3):729-47.

2. Schappert SM, Rechtsteiner EA. Ambulatory medical care utilization estimates for 2006. Natl Health Stat Rep. 2008;8:1-29. 
3. Regoli M, Chiappini E, Bonsignori F, et al. Update on the management of acute pharyngitis in children. Ital $\mathrm{J}$ Pediatr. 2011;31(37):10.

4. Shaikh N, Leonard E, Martin JM. Prevalence of streptococcal pharyngitis and streptococcal carriage in children: a meta-analysis. Pediatrics. 2010;126(3):e557-64.

5. Wade AG, Morris C, Shephard A, et al. A multicentre, randomised, double-blind, single-dose study assessing the efficacy of AMC/DCBA Warm lozenge or AMC/DCBA Cool lozenge in the relief of acute sore throat. BMC Fam Pract. 2011;18(12):6.

6. Oxford JS, Leuwer M. Acute sore throat revisited: clinical and experimental evidence for the efficacy of over-the-counter AMC/ DCBA throat lozenges. Int J Clin Pract. 2011;65(5):524-30.

7. Van Driel ML, De Sutter A, Deveugele M, et al. Are sore throat patients who hope for antibiotics actually asking for pain relief? Ann Fam Med. 2006;4(6):494-9.

8. Butler CC, Rollnick S, Pill R, et al. Understanding the culture of prescribing: qualitative study of general practitioners' and patients' perceptions of antibiotics for sore throats. Brit Med J. 1998;317(7159):637-42.

9. National Institute for Health and Clinical Excellence. NICE clinical guideline 69: respiratory tract infections-antibiotic prescribing. http://www.nice.org.uk/nicemedia/pdf/CG69Full Guideline.pdf. Accessed Mar 2013.

10. Buchholz V, Leuwer M, Ahrens J, et al. Topical antiseptics for the treatment of sore throat block voltage-gated neuronal sodium channels in a local anaesthetic-like manner. Naunyn Schmiedebergs Archiv Pharmacol. 2009;380(2):161-8.

11. American Academy of Pediatrics. Caring for a child with a viral infection. http://www.healthychildren.org/English/health-issues/ conditions/ear-nose-throat/Pages/Caring-for-a-Child-with-a-ViralInfection.aspx?. Accessed Mar 2013.

12. Berry P. Rapid relief of acute sore throat with strepsils lozenges: a single blind, comparative study. London: Royal Society of Medicine Press; 2008.

13. McNally D, Simpson M, Morris C, et al. Rapid relief of acute sore throat with AMC/DCBA throat lozenges: randomised controlled trial. Int J Clin Pract. 2010;64(2):194-207.

14. Limb M, Connor A, Pickford M, et al. Scintigraphy can be used to compare delivery of sore throat formulations. Int J Clin Pract. 2009;63(4):606-12.

15. Soldatskii YL, Onufrieva EK, Gasparyan SF, et al. Comparative effectiveness of topical antibacterial therapy of acute and relapsing chronic pharyngitis in children by means of throat lozenges and medicinal aerosol spray (in Russian). Attending Physician, Clinical Trials 2008, 1.8. http://www.lvrach.ru. Accessed Mar 2013

16. Committee for Medicinal Products for Human Use (CHMP). Reflection paper: formulations of choice for the paediatric population. EMEA/CHMP/PEG/194810/2005. http://www.ema. europa.eu/docs/en_GB/document_library/Scientific_guideline/20 09/09/WC500003782.pdf. Accessed Mar 2013.

17. Matsui D. Assessing the palatability of medications in children. Paediatr Perinat Drug Ther. 2007;8(2):55-60.

18. Pawar S, Kumar A. Issues in the formulation of drugs for oral use in children. Pediatr Drugs. 2002;4(6):371-9.
19. Hames H, Seabrook JA, Matsui D, et al. A palatability study of a flavoured desamethasone preparation versus prednisolone liquid in children with asthma exacerbation in a pediatric emergency department. Can J Clin Pharmacol. 2008;15(1):e95-8.

20. Matsui D, Barron A, Rieder MJ. Assessment of the palatability of antistaphylococcal antibiotics in pediatric volunteers. Ann Pharmacother. 1996;30:586-8.

21. Matsui D, Lim R, Tschen T, et al. Assessment of the palatability of beta-lactamase-resistant antibiotics in children. Arch Pediatr Adolesc Med. 1997;151(6):599-602.

22. Angelilli ML, Toscani M, Matsui DM, et al. Palatability of oral antibiotics among children in an urban primary care center. Arch Pediatr Adolesc Med. 2000;154(3):267-70.

23. Martinez JM, Bartyoli F, Lavanchy L, et al. A taste comparison of two different liquid colecalciferol (vitamin D3) preparations in healthy newborns and infants. Clin Drug Invest. 2006;26(11): $663-5$.

24. Bianchetti AA, Lava SA, Bettinelli A, et al. Preference for formulations containing calcium and vitamin $\mathrm{D}(3)$ in childhood: a randomized-sequence, open-label trial. Clin Ther. 2010;32(6): $1083-7$.

25. Stevens R, Votan B, Lane R, et al. A randomized study of ondansetron syrup in children: evaluation of taste acceptability and tolerance. Pediatr Hematol Oncol. 1996;13(2):199-202.

26. Tolia V, Johnston G, Stolle J, et al. Flavor and taste of lansoprazole strawberry-flavored delayed-release oral suspension preferred over ranitidine peppermint-flavored oral syrup: in children aged between 5-11 years. Pediatr Drugs. 2004;6(2):127-31.

27. Tolia V, Han C, North JD, et al. Taste comparisons for lansoprazole strawberry-flavored delayed-release orally disintegrating tablet and ranitidine peppermint-flavored syrup in children. Clin Drug Invest. 2005;25(5):285-92.

28. World Medical Association. WMA Declaration of Helsinki ethical principles for medical research involving human subjects. http://www.wma.net/en/30publications/10policies/b3/. Accessed Mar 2013.

29. Wade AG, Marshall LE, Simpson M, et al. Bioavailability and efficacy of active lozenges in the relief of sore throat pain. British Pain Society annual scientific meeting, 24-27 Apr 2007, Glasgow.

30. Sjövall J, Fogh A, Huitfeldt B, et al. Methods for evaluating the taste of paediatric formulations in children: a comparison between the facial hedonic method and the patients' own spontaneous verbal judgement. Eur J Pediatr. 1984;141(4):243-7.

31. Visser J, Kroeze JH, Kamps WA, et al. Testing taste sensitivity and aversion in very young children: development of a procedure. Appetite. 2000;34(2):169-76.

32. Boots Healthcare. Taste test of paediatric analgesic suspensions-clinical study report (BH0302), 2003 (Data on file).

33. Eccles R, Morris S, Jawad MSM. The effects of menthol on reaction time and nasal sensation of airflow in subjects suffering from the common cold. Clin Otolaryngol. 1990;15(1):39-42.

34. Bromley SM. Smell and taste disorders: a primary care approach. Am Fam Phys. 2000;61(2):427-36. 